\title{
Ein Gedanke zwei Tagungen Zum Gedenken an Marie Langer
}

Raimund Bahr (St. Wolfgang [A])

Zum 20. Todestag der aus Österreich stammenden, argentinischen Psychoanalytikerin fanden zwei Symposien der AG Literatur statt. Das eine in Zürich in Kooperation mit der Stiftung für Psychotherapie \& Psychoanalyse Zürich und das andere in Wien in Kooperation mit dem Institut für Wissenschaft und Kunst. Entstanden ist die Idee aus einem einzigen Gedanken: Marie Langer wieder in Erinnerung zu rufen. Doch die beiden Veranstaltungen hätten, obwohl ähnliche Inhalte gebracht wurden, unterschiedlicher nicht sein können. Dies hat vor allem mit der Situation der Psychoanalyse in den beiden Städten zu tun.

In Zürich hat es in den späten sechziger und siebziger Jahren eine enormen gesellschaftlichen Wandel und Aufbruch gegeben, von der auch die Psychoanalyse nicht unberührt blieb. Neben der Spaltung der Psychoanalytischen Gesellschaft, der Beteiligung der Kandidaten am internationalen psychoanalytischen Projekt «Plattform» und der Entstehung und Weiterentwicklung der Ethnopsychoanalyse stand vor allem Anfang der achtziger Jahre Nicaragua im Zentrum der Aufmerksamkeit. Marie Langer war hier durch die Arbeit vieler engagierter Menschen (vor allem Frauen) fest im psychoanalytischen und sozialpolitischen Bewusstsein präsent, vor allem durch ihre jährlichen Diskussionsveranstaltungen, die vor Ort stattfanden. Die Nähe zu Freiburg im Breisgau, wo Traute Hensch Marie Langers Bücher publizierte, tat das Übrige. Dementsprechend lebendig verlief die Veranstaltung in Zürich. Die Publikumsbeteiligung war groß und ich hatte den Eindruck, die Leute wussten, von welcher Person die Rede war. Marie Langer war hier nicht ein Mythos, sondern ein lebendige Erinnerung an einen Mensch aus Fleisch und Blut. In Zürich merkte ich, dass der gesellschaftliche Diskurs auch unter den Psychoanalytikern lebendig und kontrovers verläuft.

In Wien ging es mir seltsam. Das hat natürlich auch damit zu tun, dass ich in dieser Stadt aufgewachsen bin und vierzig Jahre dort gelebt habe. Aber obwohl die Veranstaltung im Nebenhaus der Berggasse 19 stattfand, praktisch im gleichen Haus, in dem der "Arbeitskreis für Psychoanalyse» seine Räumlichkeiten hat, war die Veranstaltung sehr gespenstisch, sehr tot. Das hat zweierlei Gründe. Einerseits gibt es öffentlich kaum einen kritischen Diskurs über den Zustand der Gesellschaft und schon gar nicht unter den Psychoanalytikern. Die öffentlichen 
Programme strotzen vor ahistorischen Studien und Fallanalysen. Andererseits ist Marie Langer hier keine lebendige Figur. Sie hat zwar formal die österreichische Staatsbürgerschaft angenommen, um während der argentinischen Diktaturen frei Reisen zu können, mehr Berührungspunkte gab es nicht, außer vielleicht infolge ihres großen Auftrittes am Internationalen Psychoanalytischen Kongress in Wien 1971. Dementsprechend verliefen auch die Diskussionen im Anschluss an die Veranstaltung. Sicher habe ich meines dazu beigetragen, dass die Diskussion schief lief, aber eine derartige Mythologisierung von Marie Langer habe ich selbst in Argentinien, wo sie sehr verehrt wird, selten zuvor erlebt.

Zusammenfassend muss ich sagen, war ich froh, dass ich das Wagnis neuerlich eingegangen bin und zwei so unterschiedliche Tagungen zu Wege brachte. Die eine (in Zürich) hat mir gezeigt, dass es nicht falsch ist, sich weiter mit Marie Langer zu beschäftigen; die andere (in Wien) hat mich darauf hingewiesen, dass es wichtig ist, dies am richtigen Ort zu tun. Und die WienerVeranstaltung hat mich darin bestätigt, dass es richtig war, diese Stadt für immer zu verlassen. 